\title{
Electron Microscopy Characterization of ZnO:Mn Nanowires
}

\author{
M. Eastman, J. Jiao
}

Department of Physics, Portland State University, Portland, OR 97201

Doping of zinc oxide nanostructures has been a popular topic of investigation to produce enhanced surface functionalization for organic materials, tuning of band gaps, [1] and altered optical properties [2] (among other things), which have direct applicability in devices such as biosensors, solar cells, and LEDs. We have evaluated the results of one such solution-based doping process by employing electron microscopy techniques.

Initially $\mathrm{ZnO}$ nanowires were grown on $\mathrm{SnO}: \mathrm{F}$-coated glass substrates. The substrates were then placed in millimolar solutions of $\mathrm{Mn}\left(\mathrm{CH}_{3} \mathrm{COO}\right)_{2}$ and $\mathrm{KOH}$ in methanol. The process was carried out at $70{ }^{\circ} \mathrm{C}$ for varied lengths of time and with differing solution conditions. The lightly doped samples endured 8-12 hours with a 1-to-1 molar solute ratio. The heavily doped wires experienced an alkaliabundant solution (10-to-1 ratio) for $24 \mathrm{hrs}$ which contributed to the over-oxidation of the nanowires (table 1). However, no residual potassium was observed in either EDS or EELS analysis.

Quantitative EDS (Table 1) reveals that there is no measurable over-oxidation of the nanowires for the light doping process (beyond the level observed in the as-synthesized nanowires). It is expected that surface $\mathrm{Zn}^{+2}$ vacancies in the nanowires provide favorable sites for attachment of $\mathrm{Mn}^{+2}$ ions in the solution. Atomic percentage of oxygen was observably reduced, though this was a less than $1 \%$ change.

HRTEM imaging and selective area diffraction (Fig. 1) of the as-grown $\mathrm{ZnO}$ nanowires show that they are highly crystalline and grow in the [0001] direction (c-axis). The lightly doped nanowires show no visible morphological changes and the crystalline quality appears to be maintained. Under the heavy doping conditions, however, surface crystallites are primarily produced and are even visible in SEM images.

Comparative EELS (Fig. 2) of the lightly and heavily doped O-K and edges shows that the concentration of Mn in the heavily doped samples is likely in the surface crystallites. Actual bulk doping may not be significantly higher than the lightly doped samples. In fact, post-annealing in these samples may tend to drive substitutional $\mathrm{Mn}^{+2}$ out of the bulk towards the surface sites made available by over oxidation. This is evident in the O-K splitting which is characteristic of oxides of manganese [3]. Also notable was that there was a measured shift of $-3 \mathrm{eV}$ in the $\mathrm{Zn}-\mathrm{L}_{3}$ edge relative to the $\mathrm{C}-\mathrm{K}$ edge (amorphous carbon) when comparing lightly doped $\mathrm{ZnO}$ to undoped (in agreement with a previously measured shift in $\mathrm{Zn} 2 \mathrm{p}_{3 / 2}$ binding energy in $\mathrm{ZnO}: \mathrm{Mn}$ measured by XPS [2]).

\section{References}

[1] R.E. Marotti et al., Solar Energy Materials \& Solar Cells 82 (2004) 85

[2] Y. Guo et al., J. Phys. Chem. C. 112 (2008) 8832.

[3] C.C. Ahn et al., EELS Atlas, Gatan, Warrendale, Pennsylvania, 1983.

[4] This research was supported in part by the National Science Foundation under awards No. CBET 0843179, ECS-0348277, and ECS-0520891. 
TABLE 1. Quantitative EDS: Undoped, doped, and heavily doped $\mathrm{ZnO}$ nanowires.

\begin{tabular}{cccc}
\hline & Undoped & Doped & Heavily Doped \\
Element & Atomic Ratio (\%) & Atomic Ratio (\%) & Atomic Ratio (\%) \\
\hline $\mathrm{Zn}$ & $44.88 \pm 0.12$ & $44.62 \pm 0.14$ & $34.42 \pm 0.24$ \\
$\mathrm{O}$ & $55.12 \pm 0.50$ & $54.98 \pm 0.52$ & $58.95 \pm 0.91$ \\
$\mathrm{Mn}$ & - & $0.40 \pm 0.07$ & $6.63 \pm 0.20$ \\
$\mathrm{Zn} / \mathrm{O}$ & $81.42 \pm 0.52$ & $81.16 \pm 0.51$ & $58.39 \pm 0.49$ \\
\hline
\end{tabular}
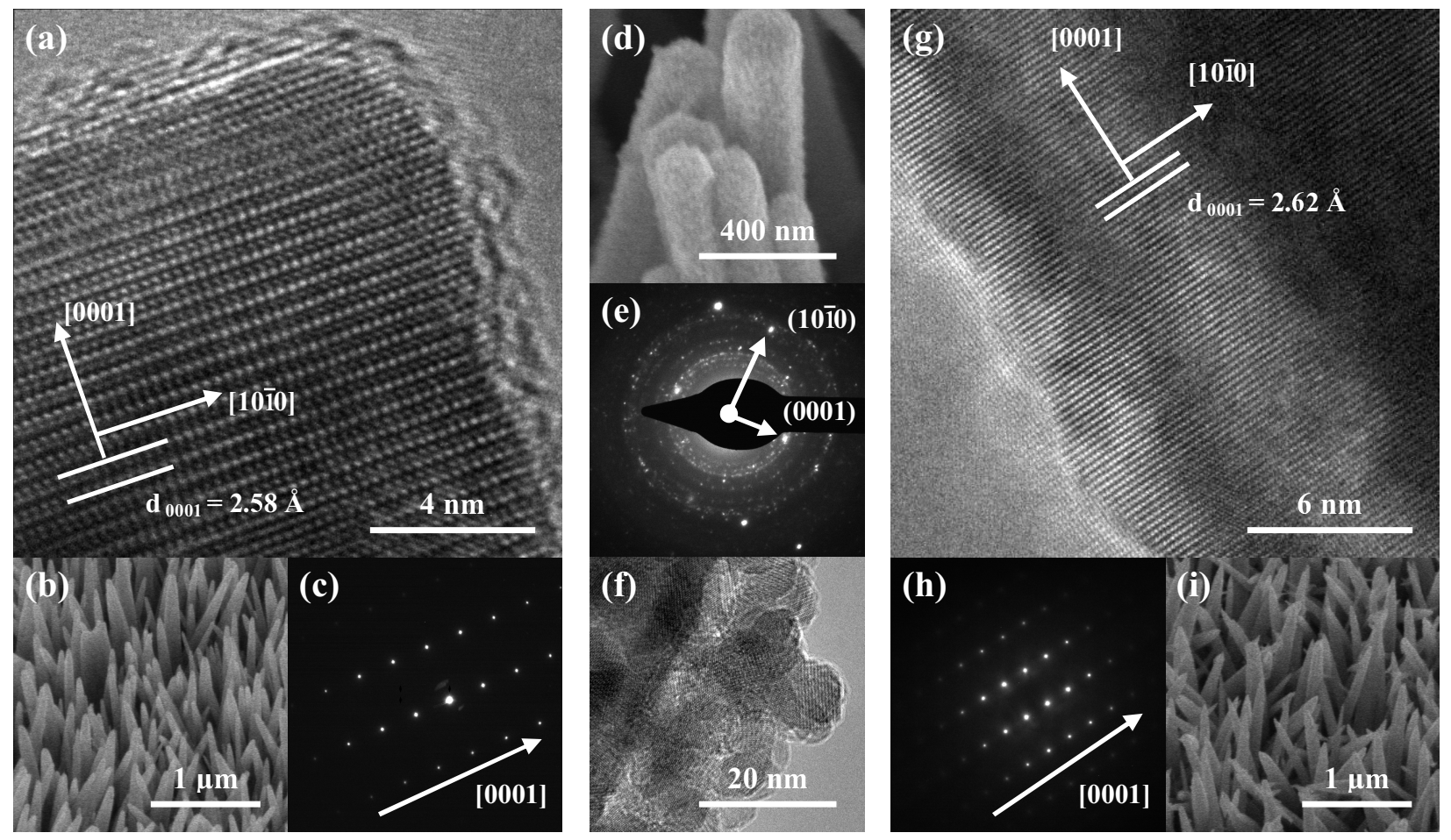

FIG. 1. HRTEM image (a), SEM image (b), and SAED (c) of undoped ZnO nanowires; SEM image (d), SAED (e), and HRTEM image (f) of heavily doped ZnO nanowires; HRTEM image (g), SAED (h), and SEM image (i) of lightly doped $\mathrm{ZnO}$ nanowires.

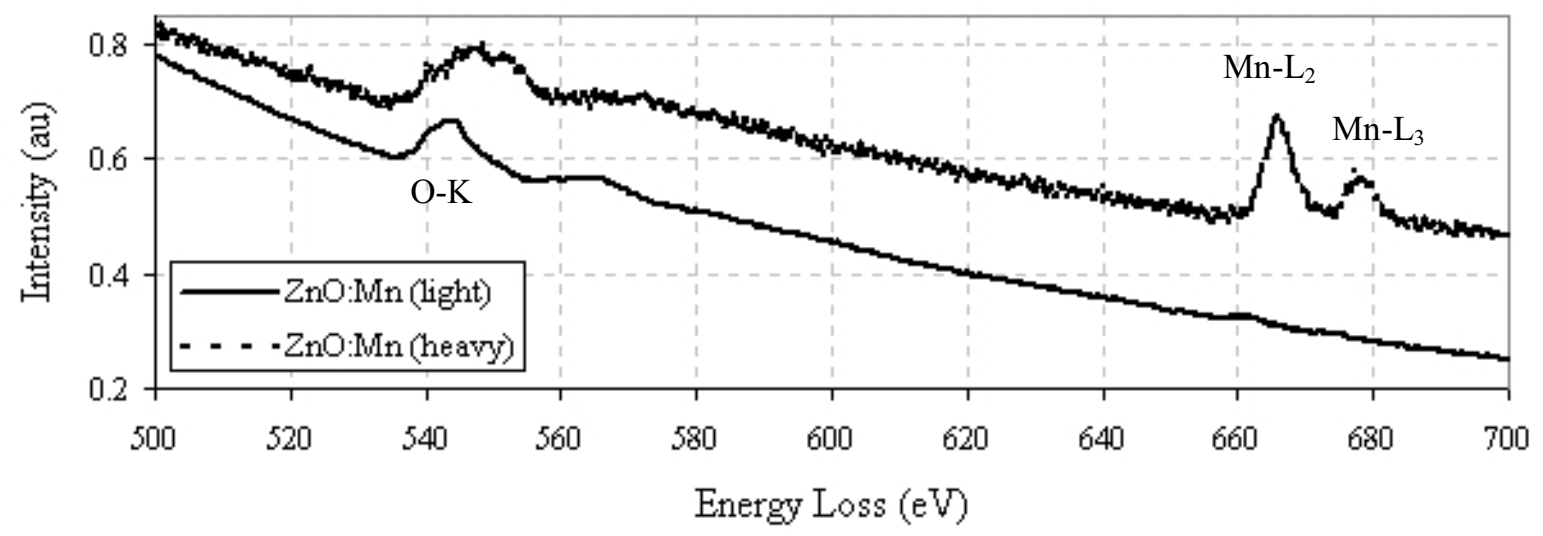

FIG. 2. Core-loss electron energy loss spectra for both lightly and heavily doped $\mathrm{ZnO}: \mathrm{Mn}$. 